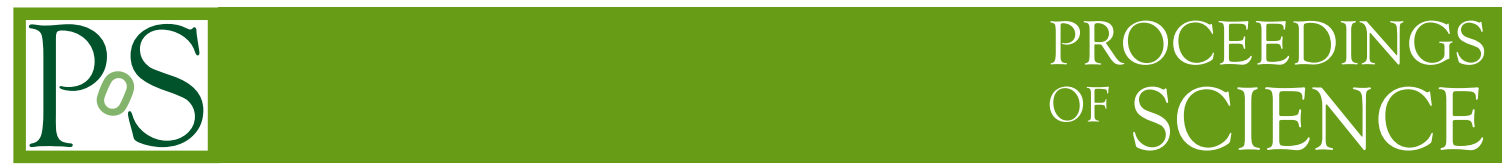

\title{
$Y(4260)$ and $\phi(2170)$ as exotic vector mesons
}

\author{
Sachiko Takeuchi*acd and Makoto Takizawa ${ }^{\text {bde }}$ \\ a Japan College of Social Work, Kiyose, Tokyo 204-8555, Japan \\ ${ }^{b}$ Showa Pharmaceutical University, Machida, Tokyo 194-8543, Japan \\ ${ }^{c}$ Research Center for Nuclear Physics (RCNP), Osaka Univ., Ibaraki, Osaka, 567-0047, Japan \\ ${ }^{d}$ RIKEN Nishina Center, Wako, Saitama 351-0198, Japan \\ e J-PARC Branch, KEK Theory Center, IPNS, KEK, Tokai, Ibaraki, 319-1106, Japan \\ E-mail: s.takeuchidjc.sw.ac.jp,takizawadac.shoyaku.ac.jp
}

The $q \bar{q} c \bar{c}$ and $q \bar{q} s \bar{s} \quad J^{P C}=1^{--}$systems are investigated by a simplified quark cluster model, where the two-meson interaction is assumed to come solely from the quark degrees of freedom. In each of the systems, one or more poles have been found. The $q \bar{q} c \bar{c}$ system seems to have a pole around the threshold with a small width. The $q \bar{q} s \bar{s}$ system has poles with rather large width. We argue that they can be seeds of the observed exotic meson candidates like $Y(4260)$ or $\phi(2170) .^{\dagger}$

XVII International Conference on Hadron Spectroscopy and Structure - Hadron2017

25-29 September, 2017

University of Salamanca, Salamanca, Spain

\footnotetext{
${ }^{*}$ Speaker.

${ }^{\dagger}$ This work was supported in part by JSPS KAKENHI Grant Number 16K05361.
} 


\section{Introduction}

In 2003, the $X(3872)$ state was first observed by Belle collaboration in $B^{ \pm} \rightarrow J / \psi \pi^{+} \pi^{-} K^{ \pm}$ [四]. It was confirmed by CDF, D0, BABAR and LHCb collaborations [2]. It has received much attention because its properties cannot be explained if one considers it as a simple charmonium. The $X(3872)$ is now considered as an exotic state. Since then, the experimentalists started searching the exotic states in the charmonium energy region and many candidates have been observed.

The $Y(4260)$ was first reported in 2005 by BABAR in the reaction $e^{+} e^{-} \rightarrow \gamma \pi^{+} \pi^{-} J / \psi$ via the initial state radiation (ISR) [3]. It was confirmed soon by CLEO [可] and Belle [5] also by ISR. The peak seems to consist of more than one resonance. Belle collaboration performed two-resonance fits in their analyses. BESIII collaboration reported recently that in the reaction $e^{+} e^{-} \rightarrow \pi^{+} \pi^{-} J / \psi$ there are three resonances in this energy region and that one of them has a mass of $4222.0 \pm 3.1 \mathrm{MeV}$ with a width of $44.1 \pm 4.3 \mathrm{MeV}$ [焑]. The average mass of $Y(4260)$ is $4230 \pm 8 \mathrm{MeV}$ with a width of $55 \pm 19 \mathrm{MeV}$ [四].

The $Y(4260)$ is considered to be an exotic meson. Its decay amplitude to $J / \psi \pi \pi$ is larger than that of a simple $c \bar{c}$ meson. It does not decay to $D \bar{D}$ states. Also, the mass of $Y(4260)$ does not fit in the theoretical $c \bar{c}$ meson mass spectrum. The decays to the exotic mesons; $Y(4260) \rightarrow$ $\pi^{\mp} Z_{c}(3900)^{ \pm}[0,[]]$ and $Y(4260) \rightarrow \gamma X(3872)[$ [Q] are observed. Theoretical approaches, such as the $c \bar{c}$ gluon hybrid model, the two-meson model, and the two-diquark model, have been taken in order to explain these features [प]]. The mechanism to make the $Y(4260)$ resonance, however, has not been understood yet. Since the final states are the hadrons, the coupling to the two-meson states should be included in the model. The nearby thresholds are the $\bar{D} D_{1}(4289.2 \mathrm{MeV}), \omega \chi_{c 1}(4293.3$ $\mathrm{MeV})$, and $\bar{D} D_{1}^{\prime}(4294.2 \mathrm{MeV})$, or $\omega \chi_{c 0}(4197.4 \mathrm{MeV})$. One may consider that $Y(4260)$ is a twomeson bound state, which couples to the two-meson states with a lower threshold and becomes a resonance. Then, the binding energy is rather large; it is about $60 \mathrm{MeV}$ below the $\bar{D} D_{1}$ threshold. The mechanism to bind this state probably contains something exotic in addition to the long range meson exchange interaction.

The $\phi(2170)\left(1^{--}\right)$has a mass of $2188 \pm 10 \mathrm{MeV}$ with a width of $83 \pm 12 \mathrm{MeV}$ []] , also referred to as $Y(2175)$. It was first observed by BaBar in 2006 also by ISR, in the $e^{+} e^{-} \rightarrow K^{+} K^{-} \pi \pi \gamma$ [ए]]. BESIII collaboration recently measured $\phi(2170)$ in the $J / \psi \rightarrow \eta \phi \pi \pi$ reaction [Ш2]. Further investigation is necessary, but it may be a s $\bar{s}$ counterpart of $Y(4260)$, and one of the candidates of the exotic mesons.

The multiquark approach can be a strong tool to investigate such exotic states: the properties of $X(3872)$ can be explained by a $1^{++} q \bar{q} c \bar{c}$ configuration which couples to the $\chi_{c 1}(2 P)$ as well as the two-meson states [[1]3, [4]]. Or, it is found that the color octet $q^{3}$ in the $u u d c \bar{c}$ configuration gives rise to a resonance around $\Sigma_{c}^{(*)} \bar{D}^{*}$ threshold, which may contribute to the LHCb pentaquark, $P_{c}$ [ए5]].

Here we propose a simplified quark cluster model, which is a quark-meson hybrid model, to investigate the $J^{P C}=1^{--} q \bar{q} c \bar{c}$ systems. The asymptotic states are two mesons, where the quark degrees of freedom are hidden in the mesons. In the short-range region, where the two mesons are close to each other, the quarks play their roles: the quark rearrangement occurs from $q \bar{q}-c \bar{c}$ to $q \bar{c}-c \bar{q}$ channels, or the the two quarks or the two antiquarks comes to interact each other. As we will show later, we have found that these features can produce resonance(s). We argue that they can be a seed 
of the $Y(4260)$ or peaks nearby. We have also applied our approach to the $J^{P C}=1^{--} q \bar{q} s \bar{s}$ systems and have found resonance(s) near $\phi(2170)$ [ए6].

\section{Model}

In this article, we investigate $J^{P C}=1^{--} q \bar{q} c \bar{c}$ and $q \bar{q} s \bar{s}$ systems to discuss the existence of such multiquark exotic mesons [16]. In the systems we consider here, the flavors of the two quarks, or those of the two antiquarks, are different from each other. So, there is no Pauli-blocking effect to consider; the quark rearrangement induces just a channel coupling between the $(q \bar{q})(c \bar{c})$ and $(q \bar{c})(c \bar{q})$ states. In the following, $c$ stands for the $s$-quark as well as the $c$-quark. And, a 'meson' corresponds to the quark-antiquark pair which is color singlet.

Among 20 kinds of $1^{--} q \bar{q} c \bar{c}$ states, 14 states correspond to the relative $S$-wave two meson states. Those are $8 q \bar{q}$-meson $c \bar{c}$-meson systems and $6 q \bar{c}$-meson $c \bar{q}$-meson systems:

$$
h_{1} \eta_{c}, f_{J} J / \psi, \eta h_{c}, \omega \chi_{c J},\left[\bar{D} D_{1}\right],\left[\bar{D} D_{1}^{\prime}\right],\left[\bar{D}^{*} D_{0}\right],\left[\bar{D}^{*} D_{1}\right],\left[\bar{D}^{*} D_{1}^{\prime}\right],\left[\bar{D}^{*} D_{2}\right] .
$$

The above states are not orthogonal to each other, because the color rearrangement factor has a nonzero value.

$$
\left\langle(q \bar{c})_{1}(c \bar{q})_{1} ; \alpha \mid(q \bar{q})_{1}(c \bar{c})_{1}, \beta\right\rangle=\sqrt{\frac{1}{9}} T_{\alpha \beta}
$$

where $(q \bar{q})_{1}$ corresponds to the color-singlet quark antiquark pair, $\alpha$ and $\beta$ are the spin-flavororbital quantum numbers, and $T$ is the spin-flavor-orbital rearrangement factor, which depends on the quark mass ratio, $m_{q} / m_{c}$, and ${ }^{t} T T=1$.

In this model, we assume the quark degrees of freedom appear only in the orbital $0 s^{2} 0 p$ configuration. The dynamical variables in the system are those of the hadrons; its potential and channel mixing come from the quark effects, which are assumed to occur only in the short range, around the hadron size. The size parameter of this $0 s^{2} 0 p$ configuration is assumed to be $x_{0} / \sqrt{m_{\text {quark }}}$, where $x_{0}$ is a constant and taken to be $0.6 \mathrm{fm}^{1 / 2}$. Namely we use a single gaussian for the orbital wave functions for the four quark states; no orbital two-quark correlation is included. By introducing the mass dependence in the size parameter in this way, however, we can include the effects that heavy quarks stay close to each other [ㅍ]].

Overlap of the model wave functions, $\mathscr{N}$, is therefore

$$
\mathscr{N}_{\alpha \beta}=\delta_{\alpha \beta}+\mathscr{P}_{0 s, \alpha}\left(N_{\alpha \beta}-\delta_{\alpha \beta}\right) \mathscr{P}_{0 s, \beta}
$$

where $\mathscr{P}_{0 s, \alpha}$ is the projection operator of the orbital $0 s$ two-meson state, and $N$ is given by

$$
N_{\alpha \beta}=\left\{\begin{array}{cl}
\delta_{\alpha \beta} & \text { both of } \alpha, \beta \text { are }(q \bar{q})(c \bar{c}) \text { or }(q \bar{c})(c \bar{q}) \\
\sqrt{\frac{1}{9}} T_{\alpha \beta} & \alpha \text { is }(q \bar{c})(c \bar{q}) \text { and } \beta \text { is }(q \bar{q})(c \bar{c})
\end{array}\right.
$$

The model Hamiltonian between the channel $\alpha$ and $\beta$ is defined as

$$
\mathscr{H}_{\alpha \beta}=H_{0}^{h} \delta_{\alpha \beta}+\mathscr{P}_{0 s, \alpha}\left(h_{\alpha \beta}^{q}-h_{0 \alpha}^{h} \delta_{\alpha \beta}\right) \mathscr{P}_{0 s, \beta},
$$


where $H_{0}^{h}$ is the free Hamiltonian for the two-meson system with the observed meson masses, $M_{1}$ and $M_{2}$, and the reduced mass $\mu=M_{1} M_{2} /\left(M_{1}+M_{2}\right)$ :

$$
H_{0 \alpha}^{h}=M_{1}^{(\alpha)}+M_{2}^{(\alpha)}+\frac{p^{2}}{2 \mu^{(\alpha)}} .
$$

In Eq. (2.5]), $h_{0}^{h}$ is a $c$-number, the matrix element of the free Hamiltonian by the two-meson in the relative $0 s$ state,

$$
h_{0 \alpha}^{h}=\sum_{i}^{4} m_{i}^{q}+\frac{3}{4 x_{0}^{2}} \times 2+\frac{5}{4 x_{0}^{2}}+\left\langle v_{\alpha}^{\text {internal }}\right\rangle=M_{1}^{(\alpha)}+M_{2}^{(\alpha)}+\frac{3}{4 x_{0}^{2}} .
$$

Here $\left\langle v^{\text {internal }}\right\rangle$ stands for $\left\langle v_{12}+v_{34}\right\rangle$ when $\alpha$ is a $(q \bar{q})(c \bar{c})$ state while it stands for $\left\langle v_{14}+v_{32}\right\rangle$ when $\alpha$ is $(q \bar{c})(c \bar{q})$ when we number the four quarks as $q_{1} \bar{q}_{2} c_{3} \bar{c}_{4}$. The potential $v$ is chosen so that the observed meson mass spectrum is reproduced.The Hamiltonian for the quarks is defined only for the $0 s^{2} 0 p$ configurations,

$$
\begin{aligned}
h_{\alpha \beta}^{q}= & \left(\sum_{i}^{4} m_{i}^{q}+\frac{3}{4 x_{0}^{2}} \times 2+\frac{5}{4 x_{0}^{2}}\right) \mathscr{N}_{\alpha \beta} \\
& +\left\langle v_{\alpha}^{\text {internal }}\right\rangle \mathscr{N}_{\alpha \beta}+\mathscr{N}_{\alpha \beta}\left\langle v_{\beta}^{\text {internal }}\right\rangle-\left\langle v_{\alpha}^{\text {internal }}\right\rangle \delta_{\alpha \beta}+\left\langle v_{13}+v_{24}\right\rangle_{\alpha \beta} .
\end{aligned}
$$

Here, $h_{\alpha \alpha}^{q}=h_{0 \alpha}^{h}$ holds because $\left\langle\alpha\left|\left(v_{13}+v_{24}\right)\right| \alpha\right\rangle=0$ for any $\alpha$. This means that the diagonal part of the model Hamiltonian $\mathscr{H}$ is free. The interaction appears only between the $(q \bar{q})(c \bar{c})$ and $(q \bar{c})(c \bar{q})$ configurations with the quark rearrangement channel coupling. The Schrödinger equation to solve now becomes the 14-channel coupled equation with the gaussian-separable potential:

$$
(\mathscr{H}-E \mathscr{N}) \Psi=0 .
$$

Parameters in the model are determined so as to reproduce the observed $q \bar{q}$ and $q^{3}$ hadron masses. The assignment of the $q \bar{q}$ mesons and their mass are summarized in Table $\mathbb{W}$. In order to construct the $q \bar{q} 0 s$ spin-spin interaction, we use the baryon mass spectrum assuming that the potential has a color $\lambda \cdot \lambda$ factor,

$$
\Delta_{u u}=\frac{1}{2}\left(2 m\left(\Sigma_{c}^{*}\right)+m\left(\Sigma_{c}\right)-3 m\left(\Lambda_{c}\right)\right)=315.2 \mathrm{MeV} .
$$

As for the $s \bar{q}$ interaction, instead of $K$ meson mass we use the mass

$$
m(s \bar{s})^{2}=2 m(K)^{2}-m(\pi)^{2}=(687.22 \mathrm{MeV})^{2} .
$$

To evaluate the size of the interaction $\left\langle v_{13}+v_{24}\right\rangle$ in Eq. ([2.5), i.e. the potential between $q$ and $c$ or $\bar{q}$ and $\bar{c}$, we uses the baryon mass spectrum,

$$
\begin{aligned}
\Delta_{u s} & =m\left(\Sigma^{*}\right)-m(\Sigma)=191.41 \mathrm{MeV} \\
\Delta_{u s}+\Delta_{u c}+\Delta_{s c} & =\frac{1}{2}\left(2 m\left(\Xi_{c}^{*}\right)-m\left(\Xi_{c}\right)-m\left(\Xi_{c}^{\prime}\right)\right)=244.38 \mathrm{MeV},
\end{aligned}
$$

and assume $\Delta_{s c}=\Delta_{u c}$ considering the mass difference between $D_{s}^{*}$ and $D_{s}$ is roughly the same size as that of $D^{*}$ and $D$. The potential between the relative $P$-wave $q$ and $c$ or $\bar{q}$ and $\bar{c}$ are taken from those of the mesons, by assuming the color $\lambda \cdot \lambda$ factor. 
Table 1: Hadron masses and their assignment. There are two $1^{+} q \bar{c}$ states, which we classified by using the total spin of the light quark $j_{q}$. Data are taken from Ref. [[]].

\begin{tabular}{cccccc}
\hline $0^{-+}$ & $1^{--}$ & $1^{+-}$ & $0^{++}$ & $1^{++}$ & $2^{++}$ \\
\hline$m_{q \bar{q}}$ & $\omega$ & $h_{1}(1170)$ & $f_{0}(980)$ & $f_{1}(1285)$ & $f_{2}(1270)$ \\
363.73 & 782.65 & 1170 & 990 & 1281.9 & 1275.5 \\
$m_{s \bar{s}}$ & $\phi(1020)$ & $h_{1}(1380)$ & $f_{0}(1500)$ & $f_{1}(1420)$ & $f_{2}^{\prime}(1525)$ \\
687.22 & 1019.460 & 1407 & 1504 & 1426.4 & 1525 \\
$\eta_{c}$ & $J / \psi$ & $h_{c}(1 P)$ & $\chi_{c 0}(1 P)$ & $\chi_{c 1}(1 P)$ & $\chi_{c 2}(1 P)$ \\
2983.4 & 3096.900 & 3525.38 & 3414.75 & 3510.66 & 3556.20 \\
\hline $0^{-}$ & $1^{-}$ & $0^{+}$ & $1^{+}\left(j_{q}=\frac{1}{2}\right)$ & $1^{+}\left(j_{q}=\frac{3}{2}\right)$ & $2^{+}$ \\
\hline$K$ & $K^{*}$ & $K_{0}^{*}(1430)$ & $K_{1}(1270)$ & $K_{1}(1400)$ & $K_{2}^{*}(1430)$ \\
495.644 & 894 & 1425 & 1272 & 1403 & 1429.0 \\
$D$ & $D^{*}$ & $D_{0}^{*}(2400)$ & $D_{1}(2430)$ & $D_{1}(2420)$ & $D_{2}(2400)$ \\
1867.21 & 2008.56 & 2318 & 2427 & 2422 & 2463.05 \\
\hline
\end{tabular}

\section{Results and Discussion}

We use the complex scaling method to solve Eq. ([2.) [ए8]. Using the values of PDG2016, there is a resonance in the $q \bar{q} c \bar{c} 1^{--}$system between $\bar{D} D_{1}$ and $\omega \chi_{c 1}$ thresholds with a width of less than $0.5 \mathrm{MeV}$. The pole, however, disappears when the assignment in Table 1 (PDG 2017 update) is used. Considering that the masses of the mesons have a certain experimental error and that the mesons have a rather large width, we made a calculation where the thresholds of $\bar{D} D_{1}, \omega \chi_{c 1}$, and $\bar{D} D_{1}^{\prime}$ to be adjusted to the same value by hand. The size of the adjustment is up to $5 \mathrm{MeV}$, and the interaction is kept unchanged. Then the resonance reappears almost on the adjusted threshold, $4293 \mathrm{MeV}$, with a width of $1 \mathrm{MeV}$. Moreover, when we assign $f_{0}(1370)$ instead of $f_{0}(980)$ to $q \bar{q}$ $0^{++}$state in evaluating the size of the potential, we found a resonance at $4445 \mathrm{MeV}$ with a width of $20 \mathrm{MeV}$.

Similar method is applied to the $q \bar{q} s \bar{s} 1^{--}$systems. There we found a pole at $2160 \mathrm{MeV}$, which is by $5 \mathrm{MeV}$ below the $K^{*} K_{1}(1270)$ threshold, with a width of $20 \mathrm{MeV}$. Since the quarkantiquark assignment to the observed mesons is not established either in this sector, we apply the same method by using the quark model meson masses [ए]]. Then we found three resonance poles: the resonance energy and the width of each of them are $2130 \mathrm{MeV}$ with $30 \mathrm{MeV}, 2260 \mathrm{MeV}$ with $70 \mathrm{MeV}$, and $2310 \mathrm{MeV}$ with $70 \mathrm{MeV}$.

The most ambiguous point of this work is that the $q \bar{q}$ and $q \bar{c}$ meson assignment. For example, both of the $f_{0}(980)$ and $f_{0}(1370)$ are considered not to be a pure $q \bar{q}$ state, but probably have $s \bar{s}$, $\pi \pi$ or $K \bar{K}$ components. The mixing of the ${ }^{1} P_{1}$ and the ${ }^{3} P_{1}$ configurations in the $K_{1}$ mesons has not been experimentally determined yet. The above poles may not stay at the same energy after such realistic mixing in the $q \bar{q} 0^{++}$or in the $1^{+}$mesons is introduced. Moreover, to discuss exotic states more realistically it is necessary to take into account the $q \bar{q}$ meson width, or the coupling to the charmonium. It is also necessary to include possible meson exchange effects such as those between $q \bar{c}$ and $c \bar{q}$ mesons. It, however, is clearly shown that the quark rearrangement and its interaction can 
cause resonance(s), which can be a seed of the observed rich spectrum around this energy region.

The authors would like to thank the audience of the session for various discussionss.

\section{References}

[1] S. K. Choi et al. [Belle Collaboration], Phys. Rev. Lett. 91, 262001 (2003)

[2] C. Patrignani et al. [Particle Data Group], Chin. Phys. C, 40, 100001 (2016) and 2017 update.

[3] B. Aubert et al. [BaBar Collaboration], Phys. Rev. Lett. 95, 142001 (2005); J. P. Lees et al. [BaBar Collaboration], Phys. Rev. D 86, 051102 (2012)

[4] Q. He et al. [CLEO Collaboration], Phys. Rev. D 74, 091104 (2006)

[5] C. Z. Yuan et al. [Belle Collaboration], Phys. Rev. Lett. 99, 182004 (2007);

[6] M. Ablikim et al. [BESIII Collaboration], Phys. Rev. Lett. 118, no. 9, 092001 (2017)

[7] M. Ablikim et al. [BESIII Collaboration], Phys. Rev. Lett. 110, 252001 (2013)

[8] Z. Q. Liu et al. [Belle Collaboration], Phys. Rev. Lett. 110, 252002 (2013)

[9] M. Ablikim et al. [BESIII Collaboration], Phys. Rev. Lett. 112, no. 9, 092001 (2014)

[10] H. X. Chen, W. Chen, X. Liu and S. L. Zhu, Phys. Rept. 639, 1 (2016) and references therein.

[11] B. Aubert et al. [BaBar Collaboration], Phys. Rev. D 74, 091103 (2006).

[12] M. Ablikim et al. [BESIII Collaboration], Phys. Rev. D 91, no. 5, 052017 (2015).

[13] M. Takizawa and S. Takeuchi, Prog. Theor. Exp. Phys. 2013, 0903 D01 (2013).

[14] S. Takeuchi, K. Shimizu and M. Takizawa, Prog. Theor. Exp. Phys. 2014 (2014) no.12, 123D01 Erratum: [ Prog. Theor. Exp. Phys. 2015 (2015) no.7, 079203]

[15] S. Takeuchi and M. Takizawa, Phys. Lett. B 764, 254 (2017).

[16] S. Takeuchi and M. Takizawa, in preparation.

[17] For example, H. Nagahiro, et al. "Structure of excited charmed baryons studied by pion emission decays," in the session of Spectroscopy of baryons, Hadron2017, Sep 29, 2017.

[18] T. Myo, Y. Kikuchi, H. Masui and K. Katō, Prog. Part. Nucl. Phys. 79, 1 (2014).

[19] S. Godfrey and N. Isgur, Phys. Rev. D 32, 189 (1985). 